\title{
SIMULATION OF DECAYS AND SECONDARY ION LOSSES IN A BETABEAM DECAY RING
}

\author{
F.W. Jones, TRIUMF, Vancouver, Canada, and E. Wildner, CERN, Geneva, Switzerland
}

\begin{abstract}
Radioactive ions injected into the decay ring of a Betabeam neutrino facility will constitute a continuous source of decay products distributed around the ring. Secondary ions from beta decays will differ in charge state from the primary ions and will follow widely offmomentum orbits. In the racetrack configuration of the ring, they will be mismatched in the long straights and may acquire large amplitudes, but the great majority of losses will be in the arcs. We describe here a comprehensive model of ion decay, secondary ion tracking, and loss detection, which has been implemented in the tracking and simulation code Accsim. Methods have been developed to accurately follow ion trajectories at large momentum deviations as well as to detect their impact coordinates on vacuum chamber walls and possibly inside magnetic elements.

Using secondary-ion data from Accsim and postprocessing with Mathematica, we have implemented a follow-on simulation in FLUKA with a 3D geometry of decay ring components and physics models for ion interactions in matter, allowing radiological studies and in particular the visualization and analysis of heat deposition in the dipole magnets which is a critical design factor for the ring. In our simulations we have also implemented absorber elements which are intended to localize the majority of losses outside of the dipoles.

These studies provide estimates of ring performance (in terms of loss concentration and management), the effectiveness of absorbers, and the implications for successful superconducting dipole operation.
\end{abstract}

\section{INTRODUCTION}

The Betabeam Decay Ring is the final stage of the accelerator complex comprising the baseline design $[1,2]$ of the EURISOL Beta Beam Task[3]. ${ }^{6} \mathrm{He}$ or ${ }^{18} \mathrm{Ne}$ ions are generated in an ISOL front end, accelerated to $\sim 100 \mathrm{GeV} / \mathrm{u}$, and ultimately injected into the racetrack structure of the decay ring, where the decays in the long straight sections give rise to well-collimated neutrino beams. The decay products ${ }^{6} \mathrm{Li}$ and ${ }^{18} \mathrm{~F}$, however, are rapidly lost from the ring and have the potential to limit its operation and maintenance. In the following we outline the path taken to study these secondary-ion losses via Monte Carlo methods, proceeding from tracking simulations to particle-in-matter simulations.

\section{DECAY PROCESS}

The multiparticle code Accsim[4] performs the first stage of modelling the histories of ions, from their injec05 Beam Dynamics and Electromagnetic Fields
Table 1: Characteristics of ions in the $\beta$ beam Decay Ring

\begin{tabular}{|r|r|c|c|c|cr|}
\hline Ion & Charge & $\gamma$ & \multicolumn{2}{|c|}{ Half-life in decay ring } & Decay product \\
& & & (sec.) & (rev.) & $\Delta p / p$ \\
\hline \hline${ }^{6} \mathrm{He}$ & +2 & 100 & 80.7 & $3.5 \times 10^{6}$ & ${ }^{6} \mathrm{Li}$ & -0.3338 \\
${ }^{18} \mathrm{Ne}$ & +10 & 100 & 167 & $7.24 \times 10^{6}$ & ${ }^{18} \mathrm{~F}$ & 0.1109 \\
\hline
\end{tabular}

tion and stacking in the decay ring, to their decay into secondary ions, and the subsequent and inevitable loss of the secondary ions from the ring, which occurs within $1 / 2$ turn of the decay location. Although Accsim can be configured to simulate the entire life cycle of ions: injection, rf stacking, pre- and post-decay tracking, and loss location, from the tracking and simulation point of view the ions have a dauntingly long lifetime in the decay ring. (see Table 1).

For the purposes of this study we have therefore considered the steady-state operation of the ring after filling or top-up has occurred, when circulating beam distributions are well-defined. We begin by macroparticle sampling from the phase space of the estimated ion distributions[6]. At the same time an additional coordinate, the ion lifetime, is sampled. Tracking is done by element transfer maps, so actual decays are detected via a master clock updated at the end of transport through each element, and then backtracked (by splitting the element transfer map) to the actual decay point inside the element. This allows the precise determination of initial conditions for each secondary ion.

\section{CHROMATIC AND GEOMETRIC EFFECTS}

The extreme mismatch of the secondary ion rigidity to the decay ring bending strength is beyond the reach of the usual fast-tracking mechanisms (e.g. matrix or thinlens maps) which are intended to deal with small deviations from the nominal ion trajectory. Relative to the reference orbit of the tracking formalism, the secondary ions are equivalent to particles that are off-momentum by $\sim 10$ $30 \%$ (see Table 1). As a simpler and much faster alternative to direct integration by ray-tracing or high-order map extraction à la COSY [5], we have employed "matrix scaling" techniques to calculate transfer maps for ions of arbitrary large momentum deviation.

For quadrupole fields we recompute the transfer matrix for each particle by scaling the focusing strength $K_{1}=$ $\left(K_{1}\right)_{0} /(1+\delta)$, where $\left(K_{1}\right)_{0}$ is the nominal quadrupole strength and $\delta=\Delta p / p$ is the fractional momentum deviation. This reproduces the (second order) chromaticity of particle tunes and also accounts for optical mismatch (beta 
beating) in which secondary ions may aquire large amplitudes in the long straights and may actually be lost before reaching the arcs.

For dipole fields it is necessary to account for both the different bending radius of the secondary ion, and its entry point to the dipole, which may be far off-center. Both these factors determine the effective length and hence the amount of bending experienced by each ion. For an ion which enters the dipole with coordinates $\left(x_{e}, x_{e}^{\prime}, \delta\right)$ we define a new off-center off-momentum reference orbit with entry coordinates as follows:

$$
x_{\mathrm{ref}}=x, \quad x_{\mathrm{ref}}^{\prime}=\left(\theta-\theta_{0}\right) / 2
$$

where $\theta_{0}$ is the nominal (on-momentum) bending angle of the dipole, and $\theta$ is the off-momentum bending angle given by

$$
\theta=2 \sin ^{-1}\left[\left(\rho_{0}+x_{\text {ref }}\right) \sin \left(\theta_{0} / 2\right) / \rho\right]
$$

where $\rho_{0}=L_{0} / \theta_{0}$ is the nominal bending radius and $\rho=$ $\rho_{0}(1+\delta)$ is the off-momentum bending radius. Since the coordinate $x^{\prime}=d x / d s$ the expression for $x_{\text {ref }}^{\prime}$ is not exact but is well approximated as all angles remain small even for the large $\delta$ of the secondary ions.

The path length along the new reference orbit is $L=\rho \theta$ and the parameters $(L, \theta)$ are used to compute a new transfer matrix along this reference orbit. The overall transfer map: translation-matrix-translation is thus customized to each particle and has been found to agree well with 8thorder COSY maps for the dipole in question.

\section{SECONDARY ION DATA: FROM ACCSIM TO FLUKA}

The design criteria of the Decay Ring superconducting dipoles[7] are strongly co-dependent with the loss pattern of secondary ions in the arcs. This loss pattern can be expressed as a series of Monte Carlo events by Accsim, where events arise from sampling of primary ions from phase space distributions, tracking of primary ions through the lattice, sampling of particle lifetimes and localization of decays, and tracking of secondary ions until they exceed the defined apertures of the ring elements.

In the present study we have used Accsim in this way as an event generator for the code FLUKA $[8,9]$ which has been extensively used in simulations of particle losses, energy deposition and heat load in the superconducting LHC dipoles[10]. For energy deposition studies we have defined a reference lattice cell: a cell in an arc of the Decay Ring which is representative of the repetitive loss pattern in the arc. This cell corresponds to a sequence of elements in Accsim which are tagged as "elements of interest" for the accumulation of secondary ion events. During Accsim tracking, events are recorded for FLUKA input according to two criteria: (1) A secondary ion (from a decay upstream) has arrived at the first element in the cell and is within the aperture of the element; (2) A primary ion decays in one of the elements of the cell. In both cases, Accsim records the event data as follows:

05 Beam Dynamics and Electromagnetic Fields
- Turn number, element number, particle number

- Event type (secondary ion or decay of primary)

- Longitudinal position ( $s$ coordinate) of event

- Transverse coordinates $x, x^{\prime}, y, y^{\prime}$

- Ion energy deviation $\Delta T$ and reference energy $T_{\text {ref }}$.

\section{HEAT DEPOSITION IN AN ARC CELL}

The events generated by Accsim are first processed by a Mathematica script which separates events by type to check the impact of particles decaying in the cell and of decayed particles tracked around the machine, escaping the absorbers. The event coordinates are transformed into the cartesian svstem of FLUKA.

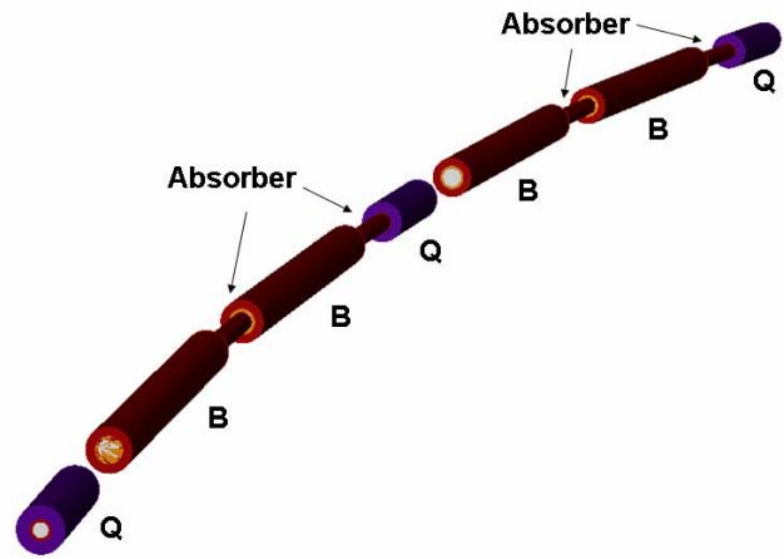

Figure 1: FLUKA geometry model of an arc cell extended with one quadrupole

The FLUKA model consists of volumes representing the $2 \mathrm{~m}$ long quadrupole, the almost $6 \mathrm{~m}$ long dipole magnets, and optionally the post-dipole absorbers (see Figure 1). For the moment no vacuum pipes are simulated. The geometry can be realised in FLUKA by individual volumes placed in the cartesian system, or by replicas of prototype volumes, that at run time are rototranslated over the prototype of the element. The latter method is preferable for energy deposition binning since the prototype is positioned in a way convenient for the postprocessing and the visualization by having the binning axes aligned to the element axes. Both models have been used and compared. The model using prototypes is also a convenient object orientated approach to enhance the magnet models later, by adapting only one prototype object.

The representative arc cell, extended with one quadrupole at the end to check the repeatability, is comprised of simplified superconducting magnet models based on preliminary magnet design studies[7]. It is important to define the volumes as well as possible, in particular around the magnet coils. We look for peak energy deposition in the coils, to evaluate the risk for quenching, and we want an overall estimation of the total power deposited in the magnet. For this estimation we use concentric cylinders corresponding to the coil and the yoke, of length corresponding to the magnetic length of the magnets. The magnetic fields[6] in the dipole and

D05 Code Developments and Simulation Techniques 
quadrupole elements are defined analytically via FLUKA user routines. Heating estimates are for the fully populated ring $\left(9.66 \times 10^{13} \mathrm{He}\right.$ or $\left.7.42 \times 10^{13} \mathrm{Ne}\right)$ in which the number of secondary ions per second deposited in the cell is $5.371 \times 10^{9}$ or $1.993 \times 10^{9}$ respectively.

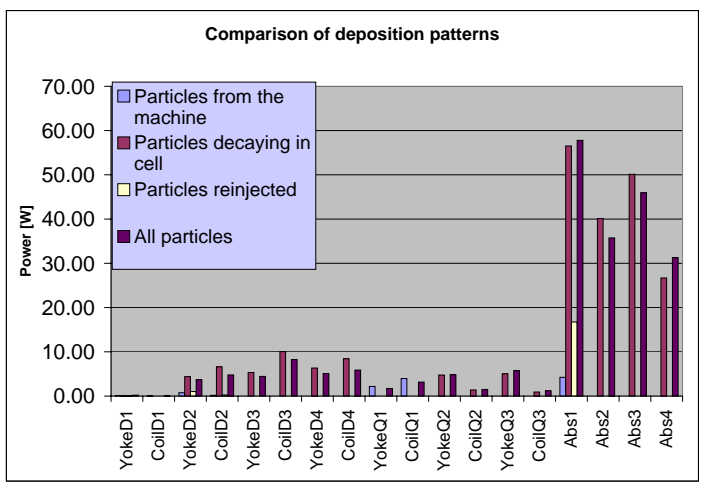

Figure 2: Heat deposition per element for ${ }^{6} \mathrm{Li}$

The FLUKA results (Figures 2 and 3) show that the heating from He decay in the dipole is $\sim 8 \mathrm{~W}$ distributed mainly over the latter $3 \mathrm{~m}$, and the Ne decay shows approximately twice this rate. These results are within the design limit of $10 \mathrm{~W} / \mathrm{m}$ longitudinally, however we observe that the peak heat deposition in the coils is concentrated in the midplane over a small region (see Figure 4) and thus the volume rate of heating for $\mathrm{Ne}$ is $12 \mathrm{~mW} / \mathrm{cm}^{3}$ which exceeds the operational limits posed by the heat transport capacity in the magnet $\left(4.3 \mathrm{~mW} / \mathrm{cm}^{3}\right.$ used for the LHC magnets).

In this simulation we have used iron absorbers of $3.5 \mathrm{~cm}$ aperture after each dipole to concentrate losses away from downstream magnets[6]. From the point of view of the impedance budget, the irregular diameter of the metallic surface that would be seen by the beam must be smoothed by a liner inside the dipole beam pipe with an opening for the horizontally escaping decay products[7], or by a beam pipe inside the absorbers, transparent to the decay.

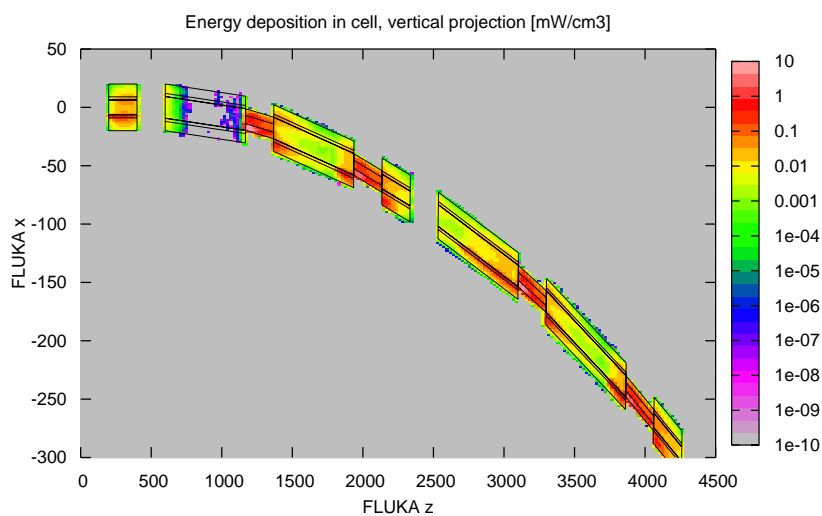

Figure 3: Energy deposition pattern in arc cell components by Lithium, with absorbers (vertical projection).

\section{CONCLUSIONS}

We have established a working protocol incorporating a large-scale accelerator tracking simulation with an ad05 Beam Dynamics and Electromagnetic Fields

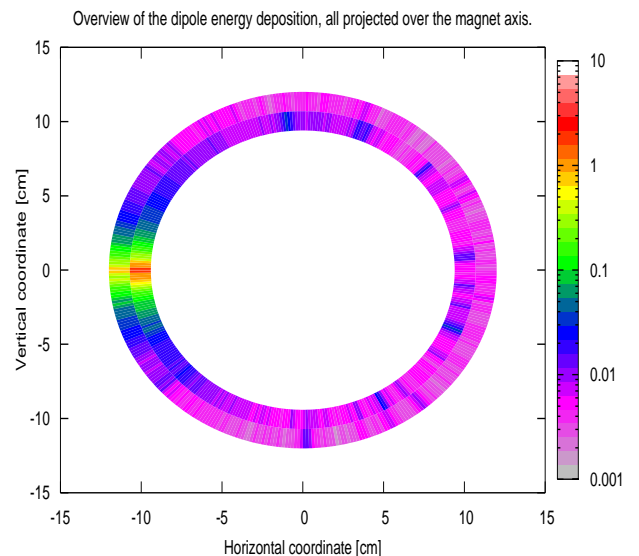

Figure 4: Energy deposition in superconducting dipole. vanced simulation of particles in matter in a full 3D geometry. The initial results for He show that the overall heat deposited in the magnets is not exceeding $10 \mathrm{~W} / \mathrm{m}$, but the local heat deposited in the second dipole is exceeding the limit accepted. For Ne the more gentle secondary ion curvature leads to greater losses in the dipole regions adjacent to the absorbers and approximately doubles the local heat deposition. Optimisation of the absorbers or a different magnet design seems necessary.

\section{ACKNOWLEDGMENTS}

We acknowledge the financial support of the European Community under the FP6 "Research Infrastructure Action - Structuring the European Research Area" EURISOL DS Project Contract No. 515768 RIDS.

We are grateful to Antoine Chancé of CEA Saclay for providing timely and accurate reference data for the current Decay Ring lattice and beam parameters, and to Francesco Cerutti of CERN for his support for the FLUKA modelling and user-routine development essential to this work.

\section{REFERENCES}

[1] M. Benedikt, S. Hancock and M. Lindroos, Baseline Design for a Beta-Beam Neutrino Facility, EPAC 2004, Lucerne.

[2] Parameter and Intensity Values, Version 2, July 2005 (docversion), EURISOL DS/TASK12/TN-05-03.

[3] http://beta-beam.web.cern.ch/

[4] F.W. Jones, Development of the ACCSIM tracking and simulation code, PAC 1997, Vancouver.

[5] M. Berz, Computational aspects of design and simulation: COSY INFINITY, Nucl. Instrum. Meth. A298:473, 1990.

[6] A. Chance and J. Payet, Studies of the injection system in the decay ring of a Beta-beam neutrino source, PAC 2005, Knoxville.

[7] E. Wildner and C. Vollinger, A large aperture superconducting dipole for "Beta Beams" to minimize heat Deposition in the coil, these proceedings.

[8] A. Fassò et al., FLUKA: a multi-particle transport code, CERN-2005-10 (2005), INFN/TC_05/11, SLAC-R-773.

[9] A. Fassò et al., The physics models of FLUKA: status and recent developments, Computing in High Energy and Nuclear Physics Conference (CHEP2003), La Jolla, 2003.

[10] C. Hoa et al., Parametric study of heat deposition from collision debris into the insertion superconducting magnets for the LHC luminosity upgrade, these proceedings.

D05 Code Developments and Simulation Techniques 